\title{
INTRODUCTION \\ Degenerative cervical myelopathy: diagnostic, assessment, and management strategies, surgical complications, and outcome prediction
}

\author{
Michael G. Fehlings, MD, PhD, ${ }^{1}$ Lindsay Tetreault, PhD, ${ }^{2}$ Patrick C. Hsieh, MD, ${ }^{3}$ \\ Vincent Traynelis, MD, ${ }^{4}$ and Michael Y. Wang, MD ${ }^{5}$
}

${ }^{1}$ Department of Surgery; ${ }^{2}$ Krembil Research Institute, Toronto Western Hospital, Toronto, Ontario, Canada; ${ }^{3}$ Department of Neurological Surgery, University of Southern California, Los Angeles, California; ${ }^{4}$ Department of Neurosurgery, Rush University Medical Center, Chicago, Illinois; and 5Department of Neurosurgery, University of Miami, Florida

$\mathrm{D}$ EGENERATIVE cervical myelopathy (DCM) is a progressive spine disease and the most common cause of spinal cord dysfunction in adults worldwide..$^{23}$ The underlying causes of DCM include static spinal cord compression by various osseoligamentous structures, dynamic injury from heightened spinal column mobility, and spinal cord tension due to abnormal spinal alignment. ${ }^{16}$ The structural changes involved in DCM include degeneration of the intervertebral discs, vertebral bodies, and facet joints; hypertrophy of the ligamentum flavum; and ossification of the posterior longitudinal ligament (OPLL). Mechanical compression of the spinal cord initiates a series of pathobiological events that can result in irreversible histological damage and neurological impairment. ${ }^{7}$ Pathophysiological mechanisms of DCM include ischemia, alterations of the vascular architecture, endothelial cell impairment, disruption of the blood-spinal cord barrier, neuroinflammation, and oligodendrocyte and neuronal apoptosis.

Patients with cord compression can present with localized pain as well as signs and symptoms of neurological dysfunction. ${ }^{23}$ Common symptoms of DCM include upperand lower-extremity weakness, paresthesias, gait impairment, decreased hand dexterity, and Lhermitte's phenomenon.

Degenerative cervical myelopathy is initially diagnosed through clinical assessment and further confirmed by advanced cross-sectional spinal neuroimaging, which often involves MRI. Imaging findings of relevance include spinal canal compromise, osseoligamentous cord compression, effacement of the cerebrospinal fluid with cord deforma- tion, T1 hypointensity, and T2 hyperintensity. ${ }^{19}$ Unfortunately, there is significant variability in the imaging techniques used to evaluate pathological features, diagnose and confirm DCM, and predict treatment outcomes.

Early reports on the natural history of the disease indicated that DCM is a relatively "benign" disorder and that patients are more likely to remain stable after disease onset than to deteriorate. ${ }^{12,17}$ In a recent systematic review of the literature, however, moderate evidence suggested that $20 \%-62 \%$ of patients with symptomatic myelopathy will deteriorate by 1 point on the Japanese Orthopaedic Association (JOA) scale 3-6 years after initial assessment. ${ }^{7}$ Treatment strategies have been designed and implemented to halt neurological deterioration and prevent disease progression and include both nonoperative and operative interventions. Several studies have indicated that patients typically do not achieve clinically significant gains $(\triangle \mathrm{JOA}$ or $\Delta$ modified [m]JOA $=0.0$ to 2.3) in function following structured nonoperative treatment. ${ }^{9,14}$ In addition, this review reported that approximately $23 \%-54 \%$ of patients convert to surgery following an initial period of conservative treatment. ${ }^{4,9}$

In contrast, evidence on the efficacy of operative intervention suggests that surgical decompression results in substantial and clinically meaningful improvements in mJOA or JOA, Neck Disability Index, and Nurick and visual analog scale scores in the short-, medium-, and longterm. ${ }^{2,18}$ With respect to surgical techniques, patients can be approached anteriorly and/or posteriorly depending on the nature of the underlying pathology, clinical presentation, sagittal alignment, and surgeon preference. ${ }^{11}$ 
Although generally safe and effective, surgery is associated with a complication rate of $14.1 \%$ (95\% CI $10.1 \%-$ 18.2\%). ${ }^{3}$ Specific types of complications include C-5 palsy $(1.9 \%, 95 \%$ CI $1.4 \%-2.4 \%)$, wound infection $(1.5 \%, 95 \%$ CI $1.0 \%-2.1 \%)$, dural tear $(1.4 \%, 95 \%$ CI $0.8 \%-1.9 \%)$, and dysphagia $(2.2 \%, 95 \%$ CI $1.4 \%-3.0 \%)$. Rates of major complications are also low: death $(0.3 \%, 95 \%$ CI $0.0 \%-$ $0.5 \%)$, stroke $(0.3 \%, 95 \%$ CI $0.0 \%-0.7 \%)$, and esophageal injury $(0.0 \%, 95 \%$ CI $0.0 \%-2.9 \%)$. Across several studies, the pooled cumulative incidence of reoperation or revision surgery was $1.4 \%$ (95\% CI $0.6 \%-2.1 \%$ ) and of adjacent segment degeneration was $1.5 \%$ (95\% CI $0.3 \%-2.7 \%){ }^{1-}$ $3,5,8,13,15,18$ Important predictors of surgical complications include an older age, a longer operative duration, a 2-stage surgery, diabetes, and a number of comorbidities. ${ }^{21,22}$ Different techniques are often associated with different types of complications: for example, 1) dysphagia is more frequent following anterior surgery, ${ }^{10} 2$ ) wound infection is more common following posterior surgery, ${ }^{3}$ and 3 ) higher rates of axial pain are observed following laminoplasty than after anterior decompression and fusion. ${ }^{6}$ According to data in a systematic review by Tetreault et al. ${ }^{22}$ however, there is only low to insufficient evidence to support differences in rates of complications across surgical techniques. Further studies are required to fill this key knowledge gap.

Accurately predicting treatment outcomes is increasingly important in a surgical setting. This task requires objective and quantitative assessment tools, a thorough neurological examination, an account of a patient's demographics and medical history, and, in some cases, imaging evaluation. Identifying important predictors of outcomes helps clinicians manage expectations, counsel concerned patients regarding potential treatment options, and develop case-specific management strategies. ${ }^{20}$ Future studies are required to determine the impact of age on outcomes and to evaluate the predictive value of several other factors, including duration of symptoms, comorbidities, disease causation, and imaging features.

This issue of Neurosurgical Focus was primarily undertaken to address some of the key knowledge gaps in the areas discussed above. The issue consists of 13 studies conducted to address key clinical questions surrounding 1) the impact of myelopathy on respiratory function, cortical activity, and risk of spinal cord injury; 2) the evaluation of DCM using various imaging techniques and quantitative assessment tools; 3) the comparative effectiveness and safety of various surgical procedures; and 4) important predictors of surgical outcomes. The issue consists of 1 narrative review, 4 systematic reviews, and 8 primary research studies.

In the first section, one study evaluates respiratory function in patients with DCM and control subjects both pre- and postoperatively. A second study assesses cortical changes that occur in myelopathy patients before and after surgery. Finally, a third study compares the risk of spinal cord injury in patients treated conservatively versus surgically for cervical spondylotic myelopathy (CSM) or OPLL.

The second section summarizes various assessment strategies for DCM. The first review discusses the normal anatomy of the cervical spine on MRI, pathological deviations from normal, MRI techniques and their reliability, and emerging imaging strategies; this summary is intended to serve as a reference for many spine care professionals when they evaluate their patients with DCM. A second review provides an overview of the psychometric properties as well as a critical appraisal of the revised and modified versions of the JOA scale; this study outlines the most suitable scale for evaluating patients from Asian and Western populations.

The objective of the third section is to compare the efficacy and safety of various surgical techniques: 1) minimally invasive posterior surgical decompression versus open anterior cervical decompression and stabilization; 2 ) anterior or posterior decompression and fusion versus decompression alone in OPLL patients; and 3) anterior corpectomy and fusion versus laminoplasty for OPLL. These studies summarize the relative risks and benefits of each technique in various populations and will enable clinicians to make evidence-based decisions on which surgical strategy to select.

The fourth section focuses on the incidence and risk factors of surgical complications following various surgical procedures, including anterior cervical discectomy and fusion, posterior fusion, laminoplasty, combined anteroposterior surgery, and central corpectomy. This information will enable surgeons to identify their high-risk patients, develop case-specific pre- and postoperative management strategies, and more accurately weigh the risks and benefits of each surgical procedure.

The fifth and final section evaluates important predictors of surgical outcomes. A meta-analysis assesses the impact of age on postoperative JOA scores, recovery rate, length of stay, and radiological outcomes. A second research article aims to identify significant predictors of achieving a minimum clinically important difference on the mJOA scale. This information will help clinicians make more informed decisions on the management of their elderly patients and enable them to predict outcomes in terms of what a patient would identify as meaningful.

This issue of Neurosurgical Focus has summarized current knowledge gaps and limitations in the evidence in order to provide direction for future investigation. These include the need to unify nomenclature and adopt the term "DCM;" to further prospectively evaluate the psychometric properties of the JOA scale and to develop other sensitive and specific measures to assess myelopathy; to outline appropriate management strategies for nonmyelopathic patients with evidence of cord compression; to better compare the efficacy and safety of various surgical techniques through either randomized controlled trials or propensity score matched analyses; and to standardize definitions of complications to enable a more accurate evaluation of treatment safety.

http://thejns.org/doi/abs/10.3171/2016.3.FOCUS16111

\section{References}

1. Bapat MR, Chaudhary K, Sharma A, Laheri V: Surgical approach to cervical spondylotic myelopathy on the basis of radiological patterns of compression: prospective analysis of 129 cases. Eur Spine J 17:1651-1663, 2008 
2. Chibbaro S, Mirone G, Makiese O, George B: Multilevel oblique corpectomy without fusion in managing cervical myelopathy: long-term outcome and stability evaluation in 268 patients. J Neurosurg Spine 10:458-465, 2009

3. Fehlings MG, Smith JS, Kopjar B, Arnold PM, Yoon ST, Vaccaro AR, et al: Perioperative and delayed complications associated with the surgical treatment of cervical spondylotic myelopathy based on 302 patients from the AOSpine North America Cervical Spondylotic Myelopathy Study. J Neurosurg Spine 16:425-432, 2012

4. Fukui K, Kataoka O, Sho T, Sumi M: Pathomechanism, pathogenesis, and results of treatment in cervical spondylotic myelopathy caused by dynamic canal stenosis. Spine (Phila Pa 1976) 15:1148-1152, 1990

5. Hirai T, Okawa A, Arai Y, Takahashi M, Kawabata S, Kato T, et al: Middle-term results of a prospective comparative study of anterior decompression with fusion and posterior decompression with laminoplasty for the treatment of cervical spondylotic myelopathy. Spine (Phila Pa 1976) 36:19401947, 2011

6. Hosono N, Yonenobu K, Ono K: Neck and shoulder pain after laminoplasty. A noticeable complication. Spine (Phila Pa 1976) 21:1969-1973, 1996

7. Karadimas SK, Erwin WM, Ely CG, Dettori JR, Fehlings MG: Pathophysiology and natural history of cervical spondylotic myelopathy. Spine (Phila Pa 1976) 38 (22 Suppl 1):S21-S36, 2013

8. Kommu R, Sahu BP, Purohit AK: Surgical outcome in patients with cervical ossified posterior longitudinal ligament: A single institutional experience. Asian J Neurosurg 9:196-202, 2014

9. Kong LD, Meng LC, Wang LF, Shen Y, Wang P, Shang ZK: Evaluation of conservative treatment and timing of surgical intervention for mild forms of cervical spondylotic myelopathy. Exp Ther Med 6:852-856, 2013

10. Kristof RA, Kiefer T, Thudium M, Ringel F, Stoffel M, Kovacs A, et al: Comparison of ventral corpectomy and platescrew-instrumented fusion with dorsal laminectomy and rod-screw-instrumented fusion for treatment of at least two vertebral-level spondylotic cervical myelopathy. Eur Spine J 18:1951-1956, 2009

11. Lawrence BD, Shamji MF, Traynelis VC, Yoon ST, Rhee JM, Chapman JR, et al: Surgical management of degenerative cervical myelopathy: a consensus statement. Spine (Phila Pa 1976) 38 (22 Suppl 1):S171-S172, 2013

12. Lees F, Turner JW: Natural history and prognosis of cervical spondylosis. Br Med J 2:1607-1610, 1963

13. Lian XF, Xu JG, Zeng BF, Zhou W, Kong WQ, Hou TS: Noncontiguous anterior decompression and fusion for multilevel cervical spondylotic myelopathy: a prospective randomized control clinical study. Eur Spine J 19:713-719, 2010

14. Matsumoto M, Chiba K, Ishikawa M, Maruiwa H, Fujimura
Y, Toyama Y: Relationships between outcomes of conservative treatment and magnetic resonance imaging findings in patients with mild cervical myelopathy caused by soft disc herniations. Spine (Phila Pa 1976) 26:1592-1598, 2001

15. Moussellard HP, Meyer A, Biot D, Khiami F, Sariali E: Early neurological recovery course after surgical treatment of cervical spondylotic myelopathy: a prospective study with 2-year follow-up using three different functional assessment tests. Eur Spine J 23:1508-1514, 2014

16. Nouri A, Tetreault L, Singh A, Karadimas SK, Fehlings MG: Degenerative cervical myelopathy: epidemiology, genetics and pathogenesis. Spine (Phila Pa 1976) 40:E675-E693, 2015

17. Nurick $S$ : The natural history and the results of surgical treatment of the spinal cord disorder associated with cervical spondylosis. Brain 95:101-108, 1972

18. Riew KD, Buchowski JM, Sasso R, Zdeblick T, Metcalf NH, Anderson PA: Cervical disc arthroplasty compared with arthrodesis for the treatment of myelopathy. J Bone Joint Surg Am 90:2354-2364, 2008

19. Tetreault L, Goldstein CL, Arnold P, Harrop J, Hilibrand A, Nouri A, et al: Degenerative cervical myelopathy: a spectrum of related disorders affecting the aging spine. Neurosurgery 77 (Suppl 4):S51-S67, 2015

20. Tetreault L, Le D, Cote P, Fehlings M: The practical application of clinical prediction rules: a commentary using case examples in surgical patients with degenerative cervical myelopathy. Global Spine J 5:457-465, 2015

21. Tetreault L, Nouri A, Singh A, Fawcett M, Nater A, Fehlings MG: An assessment of the key predictors of perioperative complications in patients with cervical spondylotic myelopathy undergoing surgical treatment: results from a survey of 916 AOSpine international members. World Neurosurg 83:679-690, 2015

22. Tetreault L, Tan G, Kopjar B, Côté P, Arnold P, Nugaeva N, et al: Clinical and surgical predictors of complications following surgery for the treatment of cervical spondylotic myelopathy: results from the multicenter, prospective AOSpine international study of 479 patients. Neurosurgery [epub ahead of print], 2015

23. Tracy JA, Bartleson JD: Cervical spondylotic myelopathy. Neurologist 16:176-187, 2010

\section{Disclosures}

Dr. Wang is a consultant for DePuy Spine, K2M, JoiMax, and Aesculap, holds a patent with DePuy Spine, and is on the medical advisory board for Vallum. Dr. Traynelis is a consultant for and patent holder with Medtronic and receives institutional fellowship support from Globus and AO. Dr. Hsieh is a consultant for Medtronic and DePuy. 